\title{
Control de Gleditsia triacanthos en el Parque Nacional Esteros de Farrapos e Islas del Río Uruguay
}

\author{
Beatriz Sosa ${ }^{\bowtie}$; Natalia Caballero; Andrés Carvajales; Gabriela Fernández; \\ Ana L. Mello \& Marcel AchKar \\ Instituto de Ecología y Ciencias Ambientales, Facultad de Ciencias, Universidad de la República, Uruguay.
}

\begin{abstract}
Resumen. Las especies invasoras constituyen una amenaza para la conservación de la biodiversidad, por lo que el desarrollo de programas para su control requiere de una evaluación cuidadosa. La invasión de Gleditsia triacanthos es una de las principales amenazas en el Parque Nacional Esteros de Farrapos e Islas del Río Uruguay. Evaluamos la efectividad del control de esta leñosa mediante la aplicación de dos herbicidas (Glifotec y Tordón) en perforaciones practicadas en el tronco, y su impacto sobre la regeneración del componente leñoso de la vegetación. Luego de dos años de la aplicación, la mortalidad media por parcela para los tratamientos con Tordón y Glifotec fue $66 \%$ y $60 \%$, respectivamente. Sin embargo, la regeneración de otras leñosas se encuentra comprometida ya que G. triacanthos representó el $92 \%$ del total de los individuos. El método de control no afectó la abundancia ni el número de géneros de las plántulas del resto de las leñosas. El desarrollo de un programa de control químico de G. triacanthos resulta viable, pero su restauración requerirá medidas de gestión activa.

[Palabras clave: control de invasoras, bosques fluviales, aplicación de herbicidas, efectos métodos de control]

Aвstract. Control of Gleditsia triacanthos in the National Park Esteros of Farrapos and Uruguay River Islands. Invasive species are one of the most important threats to biodiversity conservation. In the National Park Esteros of Farrapos and Uruguay River Islands, the invasion of Gleditsia triacanthos is one of the main threats. We evaluated the feasibility of implementing a control program of G. triacanthos by drilling the stem and applying herbicide. We characterized the regeneration pattern of the woody component and analyzed the herbicide impact on the abundance and number of genus of woody seedlings. The herbicides used were Glifotec and Tordon. After two years of herbicide application the average mortality was $66 \%$ and $60 \%$ in Glifotec and Tordon, respectively. The regeneration of the woody component is threatened as G. triacanthos accounted for $92 \%$ of the individuals. Control did not affect the abundance and richness of seedlings of the woody component. The development of chemical control of G. tiracanthos in the National Park Esteros Farrapos is feasible but requires active restoration management.
\end{abstract}

[Keywords: invasions management, riparian forests, herbicides, effects of control methods]

\section{INTRODUCCIÓN}

Las invasiones biológicas constituyen uno de los principales determinantes de la pérdida de diversidad biológica y de la alteración de la capacidad de los ecosistemas para proveer servicios ambientales (Vitousek et al. 1997). Gleditsia triacanthos es una especie leñosa perteneciente a la familia de las fabáceas, originaria de América del Norte, y se la considera invasora en Argentina y Uruguay (Chaneton et al. 2004; Nebel \& Porcile 2006). El Parque Nacional Esteros de Farrapos e Islas del RíoUruguay (PNEF) constituye el humedal fluvial más representativo del Uruguay, y $G$. triacanthos está presente en 600 ha $(10 \%$ de la superficie del parque). Domina numerosas áreas del bosque nativo, su frente de avance también incluye ambientes de pradera y pajonal, y tiende al reemplazo casi total de

Editora Asociada: María Semmartin

$\triangle$ beatriz@fcien.edu.uy las especies nativas (DINAMA 2014). En este marco resulta relevante evaluar la efectividad del método de control para implementar un programa de manejo.

Tradicionalmente, a Gleditsia triacanthos se la controla mediante métodos químicos. En Argentina se utiliza el herbicida Togar BT (Di Marzio et al. 2009), mientras que en Uruguay se aplica glifosato, previo anillado del tronco. La perforación del tronco y posterior aplicación de herbicidas constituye la medida de manejo más adecuada ya que minimiza los efectos sobre el entorno, reduce el impacto visual y disminuye el tiempo y los costos de operación. Su aplicación en G. triacanthos fue evaluada con resultados positivos (Blumetto 2010), pero su efectividad debe ser analizada al menos dos años después de la aplicación, dada su capacidad de rebrote (Stanley et

Recibido: 29 de agosto de 2014, Fin de arbitraje: 5 de diciembre de 2014, Última versión revisada: 11 de septiembre de 2015, Aceptado: 19 de septiembre de 2015. 
al. 2014).Para implementar un programa de manejo de especies invasoras deben evaluarse los efectos de los métodos de control sobre las comunidades y las especies que se intenta proteger (Byers 2002), evaluándose la regeneración de la especie invasora y las posibilidades de restauración autogénica de la comunidad nativa. G. triacanthos presenta una regeneración elevada debido a su abundante producción de semillas y a su gran capacidad de germinación (Marco \& Páez 2000). Planteamos que en el PNEF, el control de adultos resultará viable, pero que el recambio con especies nativas requerirá el manejo de los estratos emergentes y, eventualmente, la replantación de especies nativas. En este marco se plantearon dos objetivos: 1) evaluar los efectos de la aplicación de herbicidas con perforación en individuos adultos de G. triacanthos, y 2) caracterizar el patrón de regeneración del componente leñoso.

\section{MAteriales y MÉTOdos}

\section{Sistema de estudio}

El PNEF pertenece al Sistema Nacional de Áreas Protegidas de Uruguay. Está ubicado sobre la costa del Río Uruguay (32³7'36.308" S - 5809'40.920" O). Abarca 5760 ha de bosque fluvial, praderas con régimen de inundación semipermanente, áreas paludosas, lagunas y arenales fluviales. Las principales actividades productivas son la ganadería, la pesca y la apicultura.

El área de estudio comprende 1.5 ha de bosque fluvial. Constituye un ambiente de características hidromórficas y topografía plana con variaciones a nivel del microrelieve que generan diferencias en las condiciones edáficas. G. triacanthos es una de las especies dominantes del área. Es polígamo dioica o monoica. Su fruto es una legumbre linealcomprimida. Presenta semillas elipsoidales u ovales inmersas en el mesocarpio algo pulposo y dulce, muy duras y lisas (Sabattini et al. 2009).

\section{Diseño experimental}

Realizamos un ensayo en siete bloques, con tres parcelas de $20 \mathrm{~m}^{2}(2 \times 10 \mathrm{~m})$ : dos para los tratamientos con herbicidas y una como control (sin perforación y sin herbicida). Los bloques constituyeron unidades homogéneas definidas en el terreno. El ganado vacuno mantuvo el acceso a las parcelas durante los años de las mediciones.

Se perforó el tronco de todos los individuos de G. triacanthos con más de $2 \mathrm{~m}$ de altura, ubicados dentro de las parcelas, para lo que se usó una mecha de $15 \mathrm{~mm}$. Las perforaciones se realizaron en la circunferencia a la altura del pecho, a razón de una perforación por cada $5 \mathrm{~cm}$ de diámetro del árbol, con una profundidad de $10 \mathrm{~cm}$ y una inclinación aproximada de $45^{\circ}$. El tratamiento se aplicó en noviembre de 2011 a un total de 108 árboles, durante la fase de mayor actividad fisiológica y sensibilidad (Tu et al. 2001). Los herbicidas utilizados fueron Tordón y Glifotec, de uso extendido en Uruguay. El principio activo del Tordón es derivado del ácido piridiloxiacético (Picloram) y fenoxiacético (2,4-D). Es un simulador de la hormona auxina que estimula el crecimiento tisular desorganizado (Tu et al. 2001). Su clasificación toxicológica es "moderadamente peligroso". El herbicida, Glifotec tiene como principio activo la sal isopropilamina de glifosato, que impide la síntesis de aminoácidos requeridos para la síntesis de proteínas (Tu et al. 2001). Su clasificación toxicológica es "moderado". Se preparó una solución al 50\% en agua, y se aplicó la dosis recomendada comercialmente por unidad de superficie. El volumen de la inyección fue de 17 $\mathrm{cm}^{3}$, lo que corresponde a una dosis de $4.1 \mathrm{~g}$ de sal isopropilamina de glifosato para los tratamientos con Glifotec, a una dosis de $0.1 \mathrm{~g}$ de Picloram y a $0.4 \mathrm{~g}$ de fenoxiacético $(2,4-\mathrm{D})$ para los tratamientos con Tordón las perforaciones fueron selladas con masilla luego de la aplicación del herbicida. Se registró el diámetro a la altura de pecho de los individuos tratados.

El efecto de los tratamientos fue registrado dos años después de la aplicación (febrero 2014). Se consideraron muertos a los individuos sin actividad vegetativa en el dosel al momento del registro. Se comparó la mortalidad registrada en cada tratamiento calculando la mortalidad como el cociente entre los individuos muertos y el total de individuos tratados en cada parcela. Los datos cumplieron con el criterio de homocedasticidad, por lo que el efecto del tratamiento sobre la mortalidad se evaluó mediante un análisis de varianza de efectos fijos para los factores tratamiento y bloque. Las comparaciones de medias se realizaron utilizando la prueba de Tukey. Se evaluó la relación existente entre la mortalidad y el DAP de los individuos de G. triacanthos utilizando curvas de regresión logística (McDonald 2009). La mortalidad fue considerada como variable dicotómica, y asumió valores de 1 y 0 para los individuos muertos y vivos, respectivamente, mientras que el DAP fue tratado como variable continua.

En el verano de 2013 se registró la abundancia de todas las plántulas (individuos leñosos con una altura $\leq 25 \mathrm{~cm}$ ) de $G$. triacanthos presentes en las parcelas. En el verano de 2014 se registró la abundancia de todas las plántulas presentes en las parcelas y se las identificó a nivel de género. Además, se registró la abundancia de los individuos de G. triacanthos entre 1 y $2 \mathrm{~m}$. Los individuos entre 0 y $25 \mathrm{~cm}$ representan individuos con menos de dos años para detectar efectos de los herbicidas; los individuos de G. triacanthos entre 1 y 2 m indican el potencial de desarrollo de esta especie en las parcelas tratadas. La abundancia se estimó como número de individuos por parcela $\left(20 \mathrm{~m}^{2}\right)$. Se analizó la variación interanual (2013-2014) del efecto de los tratamientos sobre la abundancia de 
plántulas de G. triacanthos mediante un análisis de varianza con medidas repetidas y dos factores. Los datos cumplieron con la hipótesis de esfericidad por lo que se utilizó el estadístico F sin corrección (von Ende 1993). Se evaluó el efecto de los herbicidas sobre la abundancia y el número de géneros de especies leñosas (excluyendo G. triacanthos). Los datos presentaron homocedasticidad. Para evaluar el efecto se utilizó análisis de varianza de efectos fijos.

\section{Resultados}

La aplicación de herbicida controló de forma eficiente a los individuos adultos de $G$. triacanthos. Dos años después de su aplicación, la mortalidad media por parcela para los tratamientos con Tordón y Glifotec fue 0.66 y 0.6 , respectivamente, mientras que en los controles no se registró mortalidad (Figura 1). La mortalidad entre herbicidas fue similar $(P=0.9)$ (Figura 1). El DAP promedio de los individuos tratados fue $13+7 \mathrm{~cm}$, y los valores mínimos y máximos registrados fueron 5 y 40 $\mathrm{cm}$, respectivamente. La mortalidad no tuvo relación con el DAP (Tordón: $\chi_{(1)}^{2}=1.5 ; P=0.2$; Glifotec: $\left.\chi_{(1)}^{2}=1 ; P=0.3\right)$.

La regeneración de leñosas estuvo dominada por individuos de G. triacanthos que representaron $92 \%$ y $91 \%$ de los individuos entre $0-25 \mathrm{~cm}$ de talla en los años 2013 y 2014, respectivamente. En el año 2014 se identificaron dos estratos: el primero, conformado por plántulas de hasta $25 \mathrm{~cm}$, y el segundo, con individuos cuya altura varía entre 1 y 2 m (Tabla 1). Cabe destacar que la abundancia en el estrato de $0-25 \mathrm{~cm}$ fue mucho mayor a la del estrato comprendido entre 1 y $2 \mathrm{~m}$, cuya abundancia promedio fue menor a 1 (Tabla 1). La abundancia de plántulas de G. triacanthos no varió entre los dos períodos analizados $(P=0.6)$, y tampoco se detectó un efecto de los tratamientos $(P=0.8)$ o de la interacción entre estos factores $(P=0.8)$.

Tabla 1. Abundancia de Gleditsia triacanthos (individuos/ $20 \mathrm{~m}^{2}$, promedio $\pm 1 \mathrm{DS}$ ) en los estratos $0-25 \mathrm{~cm}$ y $1-2 \mathrm{~m}$. Abundancia y número de géneros de las especies leñosas en el estrato 0-25. Datos registrados en febrero de 2014.

Table 1. Gleditsia triacanthos' abundance (individuals $/ 20$ $\mathrm{m}^{2}$, average $\pm 1 \mathrm{SD}$ ) in the $0-25 \mathrm{~cm}$ and $1-2 \mathrm{~m}$ strata. Abundance and number of genus of woody species. Data was registered in February 2014.

\begin{tabular}{|c|c|c|c|c|}
\hline & \multicolumn{2}{|c|}{ Gleditsia triacanthos } & \multicolumn{2}{|c|}{ Otras leñosas } \\
\hline & $\begin{array}{l}\text { Abundancia } \\
(0-25 \mathrm{~cm})\end{array}$ & $\begin{array}{l}\text { Abundancia } \\
\quad(1-2 \mathrm{~m})\end{array}$ & $\begin{array}{l}\text { Abundancia } \\
(0-25 \mathrm{~cm})\end{array}$ & Géneros \\
\hline Tordón & $86 \pm 77$ & $0.2 \pm 0.4$ & $6.4 \pm 4.4$ & $2 \pm 1$ \\
\hline Glifotec & $81 \pm 43$ & 0 & $8.1 \pm 8.9$ & $3 \pm 2$ \\
\hline Control & $86+38$ & $0.7 \pm 1.3$ & $8 \pm 9$ & $3 \pm 2$ \\
\hline
\end{tabular}

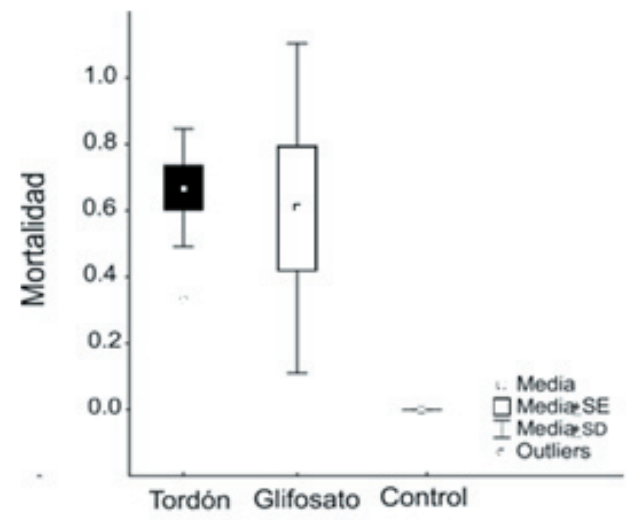

Figura 1. Efectos de la aplicación de Tordón y Glifotec sobre la mortalidad de Gleditsia triacanthos. Mortalidad $=$ nro. de individuos muertos/nro. de individuos tratados.

Figure 1. Herbicide effects (Tordón and Glifotec) on Gleditsia triacanthos' mortality. Mortality = no. of dead individuals/no. of treated individuals.

Además de G. triacanthos, en el estrato emergente se registraron 9 géneros de especies leñosas, que representaron el $8 \%$ de los individuos de hasta $25 \mathrm{~cm}$. En cambio, no se registraron individuos en el estrato de entre 1 y $2 \mathrm{~m}$. Los géneros nativos identificados fueron Sebastiania, Blepharocalyx, Erythrina, Maytenus, Eugenia y Myrceugenia. Se registraron dos géneros reconocidos en Uruguay como exóticos: Morus y Laurus, y uno exótico invasor, Fraxinus, que fue el más abundante del estrato con casi la tercera parte de los registros. No se detectó efecto de los tratamientos sobre la abundancia en el estrato emergente $(P=0.5)$, pero sí entre bloques $(P<0.0001)$ (Tabla 1$)$. No se identificaron diferencias significativas del número de géneros vinculadas a los tratamientos $(P=0.3)$, pero sí entre bloques $(P<0.0001)$ (Tabla 1).

\section{DISCUSIÓN}

Luego de dos años de implementado, el método de control con perforación de tronco e inyección de herbicida parece apropiado para el control de G. triacanthos. Este método resulta pertinente ya que reduce los daños en el ambiente en relación a la metodología tradicional de control, basada en el anillado y posterior aplicación de herbicida (reconocido como de alto riesgo ecotoxicológico [Di Marzio et al. 2009]).

El tipo de herbicida no afectó la mortalidad. Si bien con esta técnica se minimizan los impactos sobre el entorno, el herbicida Tordón presenta un mayor nivel de toxicidad, por lo que el uso de Glifotec resulta más apropiado. 
De hecho, es uno de los más utilizados en áreas naturales (Tu et al.2001). Las aplicaciones de herbicida se realizaron utilizando las dosis comerciales, pero para optimizar la eficacia del método se debe estudiar la curva dosisrespuesta a nivel de aplicación en individuos. También es necesario identificar la dosis que minimice el número de perforaciones $\mathrm{y}$, por lo tanto, el tiempo de operación. En este trabajo se realizó una perforación cada $5 \mathrm{~cm}$ de DAP del árbol, la mitad de las perforaciones recomendadas (Tu et al. 2001). Los resultados fueron muy buenos.

La mortalidad de individuos no se relacionó con el DAP, lo que sugiere que la dosis aplicada (ajustada según el tamaño del árbol) fue efectiva para todas las tallas. Santos \& Monteiro (2007) encontraron un incremento significativo en la mortalidad con el incremento del área seccional para Acacia dealbata y A. Melanoxylon, y una relación inversa entre la mortalidad y el área seccional para Eucalyptus globulus cuando fueron tratadas con la misma dosis de glifosato. Estos resultados sugieren que la relación entre el tamaño del árbol y la mortalidad estaría determinada por la respuesta particular de cada especie a la aplicación del herbicida. Esta respuesta debería ser estudiada para $G$. triacanthos a fin de optimizar la técnica. La profundidad de las perforaciones realizadas fue $10 \mathrm{~cm}$, excediendo el área de tejido vivo susceptible a la aplicación de herbicida. Reducir la profundidad de las perforaciones disminuiría el tiempo de operación.

Gleditsia triacanthos dominó el estrato en regeneración con el 90\% del total de los individuos. Sin embargo, su abundancia en el estrato 1-2 m fue muy baja, lo cual indica que su desarrollo posterior se encuentra limitado. Estos resultados podrían explicarse por la ganadería, que facilitaría el establecimiento de plántulas mediante dispersión y escarificación de las semillas (USDA 2014), pero afectaría su crecimiento debido al pastoreo. En tal sentido, el efecto neto de la ganadería sobre el desarrollo de G. triacanthos estará determinado por la magnitud del efecto de estos procesos que operan en sentidos contrarios. Cabe destacar que en bosques ribereños de la Pampa Ondulada el efecto de la ganadería fue reportado como facilitador del desarrollo de G. triacanthos (Leggieri 2010).

El régimen de inundaciones del humedal podría afectar el crecimiento de G. Triacanthos, así como su dispersión. En el período de esta investigación se registraron al menos
44 días en los que el nivel del agua fue $\geq 3$ m.s.n.m. Durante estos eventos, las plántulas quedan sumergidas por completo. El estrés fisiológico asociado a los eventos podría reducir su desarrollo e incluso determinar su mortalidad.

La abundancia de G. triacanthos en el estrato de hasta $25 \mathrm{~cm}$ no varió entre años ni fue afectada por el tipo de tratamiento. Esto indica que en las parcelas estudiadas, el establecimiento de plántulas no fue afectado por la mortalidad de los individuos adultos. Estos resultados no coinciden con las observaciones de Marco \& Páez (2000), quienes encontraron que la mortalidad de adultos aumenta la disponibilidad de luz en los estratos inferiores, lo cual favorece el desarrollo de plántulas y/o de juveniles de G. triacanthos. En el área de estudio, la abundancia elevada de plántulas registrada en los controles sugiere que las condiciones ambientales no limitarían su desarrollo. Alternativamente, la mortalidad de adultos reduciría el aporte de semillas en las parcelas y, en concomitancia, el desarrollo de plántulas. Así se compensa una posible facilitación por el incremento en la disponibilidad de luz. No obstante, en el área de estudio se puede esperar un aporte importante de propágulos desde las áreas cercanas por la ganadería y la hidrocoria, mecanismo de dispersión típico en humedales (van den Broek et al. 2005). Además, G. triacanthos presenta una alta viabilidad en el banco de semillas (USDA 2014), lo que podría enmascarar una reducción en el aporte de propágulos en la ventana temporal (dos años) de este estudio.

No se detectaron efectos de los tratamientos sobre la abundancia y el número de géneros del componente leñoso (excluyendo a $G$. Triacanthos), por lo que no afectarían la regeneración del bosque (a pesar de sus posibles modificaciones en variables que afectan la germinación y el desarrollo como la disponibilidad de luz y la temperatura). El período temporal analizado, dos años, podría resultar insuficiente para detectar efectos de los tratamientos sobre la regeneración de la comunidad leñosa, ya sea porque las condiciones ambientales no se afectan significativamente o porque la dinámica de regeneración responde más lentamente. Alternativamente, la capacidad de detección de estos efectos podría haberse mejorado incluyendo los datos iniciales de densidad como covariable en el análisis de varianza, reduciendo así el error experimental. En 
resumen, si bien los resultados indican que los tratamientos no afectan la regeneración de la comunidad leñosa, sí podrían detectarse en una escala temporal más extensa. Este estudio sugiere que resultaría viable implementar un programa de control para G. triacanthos, pero que la restauración requerirá monitoreo y medidas de gestión activa.

Agradecimientos: A R. Merni, F. Mendieta, G. Viera y D. Jaso por su apoyo en las tareas de campo e interpretación de los resultados

\section{BIBLIOGRAFÍA}

Blumetto, O. 2010. Evaluación de métodos de control de especies exóticas invasoras en monte nativo. Seminario Biodiversidad. Conservación y Usos Sostenible de la Biodiversidad. Maldonado, Uruguay. www.cebra.com.uy/ presponsable/adjuntos/2010/04/oscar-blumetto-1.pdf.

BYers, JE; S REICHARD; JM RANDALl; IM PARKER \& C SMITH. 2002. Directing Research to Reduce the Impacts of Nonindigenous Species.Conserv Biol, 16:630-640.

Chaneton, EJ; CN Mazia; M Machera; A Uchitel \& C GHERSA. 2004. Establishment of Honey Locust (Gleditsia triacanthos) in Burned Pampean Grasslands. Weed Technol, 18:1325-1329.

Di Marzio, W; ME SÁenz; J Alberdi; N Fortunato; M TANGORRA; ET AL. 2009. Estrategia de manejo de acacia negra (Gleditsia triacanthos) en la cuenca del río Luján. Evaluación ecotoxicológica del herbicida Togar BT. Revista Argentina de Ecotoxicología y Contaminación Ambiental, 1:1-7.

DINAMA. 2014. Plan de Manejo del Parque Nacional Esteros de Farrapos e Islas del Río Uruguay. Dirección Nacional de Medio Ambiente. División Sistema Nacional de Áreas Protegidas. Uruguay.

LEGGIERI, LR. 2010. Invasión de Gleditsia triacanthos en los corredores de los sistemas fluviales de la Pampa Ondulada y su efecto sobre la distribución de Myocastorcoypus. Ecología Austral, 20:201-208.

MArco, DE \& SA PÁez. 2000. Invasion of Gleditsia triacanthos in Lithraeaternifolia Montane Forests of Central Argentina. Environ Manage, 26:409-419.

McDonald, JH. 2009. University of Delware. SegundaEdición. Handbook of Biological Statistics.Sparky House Publishing Baltimore, Maryland, USA. Pp. 313.

Nebel, J \& J Porcile. 2006. La contaminación del Bosque Nativo por especies arbóreas y arbustivas exóticas. www.guayubira.org.uy/monte/Contaminacion_ monte_nativo_exoticas.pdf.

Sabattini, RA; S Ledesma; E Fontana \& JM Diez. 2009. Revisión crítica de "Acacia Negra" Gleditsia triacanthos, Leñosa invasora de los sistemas productivos en Argentina. Dow AgroSciences www.dowagro.com/ar/ lineadepasturas/trabajos/acacia_negra.htm.

Stanley, W; S Zedaker; J Seiler; \& P Burch. 2014. Methods for Rapid Screening in Woody Plant Herbicide Development. Forests, 5:1584-1595.

Tu, M; C Hurd \& JM Randall. 2001. The Nature Conservancy. Weed Control Methods Handbook. tncweeds.ucdavis.edu (version abril 2001).

United States Department of Agriculture (USDA). Natural Resource Conservation Service. 2014. Plant Guide. HoneyLocust. Gleditsia triacanthos. plants.usda.gov/plantguide/pdf/pg_gltr.pdf.

van Den BRoek, T; R van Diggelen \& R BobBink. 2005. Variation in seed buoyancy of species in wetland ecosystems with different flooding dynamics. Journal of Vegetation Science, 16:579-586.

VitouseK, PM; HA MoOnEY; J Lubchenco \& JM Melillo. 1997. Human Domination of Earth's Ecosystems. Science, New Series, 277:494-499.

VONENDE, CN. 1993. Repeated-measures analysis: Growth and other time-dependent measures. Chapter 6. Pp. 113137 en: Scheiner, SM \& J Gurevitch (ed.). Design and analysis of ecological experiments. Chapman \& Hall, New York. 